\title{
Analysis of Optical Propagation in Thick Holographic Gratings
}

\author{
R. Alferness \\ Electro-Optics Lab, Dept. of ECE, University of Michigan \\ Ann Arbor, Mich. 48104, USA \\ Received 20 November 1974/Accepted 24 January 1975
}

\begin{abstract}
A method of analyzing optical propagation in thick holographic gratings by decomposition of the thick material into thin gratings is discussed. The method is readily applicable to study propagation in multiple gratings of arbitrary spatial frequency and orientation recorded in the same thick emulsion. Applied to the double grating case, the method predicts strong cross-coupling between the two gratings for proper relative slope of the gratings. Results are given.
\end{abstract}

Index Headings: Thick holographic gratings -Optical propagation

The theory of optical propagation in a holographically produced grating recorded in a thick emulsion has been developed by several authors $[1-4]$. When valid, Kogelnik's closed form solution using the coupled-wave approach is perhaps the most useful [2]. However, the method requires strong Bragg effects so that only the zero order and the one strong diffracted wave need be considered. The matrix theory developed by Burckhardt [3] and Kaspar [4], which does not require the assumptions made by Kogelnik and is theoretically capable of providing exact results, including the energy exchange among higher orders, requires finding the eigenvalues and eigenvectors of an infinite matrix by means of a digital computer. In practice the matrix must be truncated, but the error introduced is small. Kogelnik has included the possibility of sloped fringes while the latter two assume that the fringes are perpendicular to the plane of the emulsion. Neither of these methods applies to a grating structure composed of two or more sets of fringes of arbitrary spatial frequency and orientation recorded in the same emulsion. Such gratings are complicated by the possibility of light diffracted from one fringe structure subsequently being diffracted by a different set of fringes.

We describe here a new, intuitively simple method for analyzing optical propagation in general grating structures holographically recorded in a thick emulsion. The approach is particularly well suited to treat several sinusoidal fringe structures of arbitrary frequency and orientation recorded in the same emulsion. Even for the single grating case, the conceptual simplicity and ease of implementation of this approach offers advantage over the theories given by Burckhardt and Kaspar. While, like the matrix theory, the method is numerical, it requires only matrix multiplication. The method proceeds by decomposing the thick material into a series of thin slabs, each of which acts simply as a thin grating. Very general applicability can be obtained because all that need be specified are the properties of the thin gratings which may be absorption, phase or mixed. Because the effect of higher orders is included, exact results are possible and there is no need to require strong Bragg effects. Multiple fringe structures can be considered by allowing each thin grating to have several basic spatial frequencies and appropriate fringe slant factors.

\section{Deseription of the Method}

For simplicity we describe the method for a single grating structure although extension to the multiple grating case is straightforward. We consider the grating produced holographically, as shown in Fig. 1. 


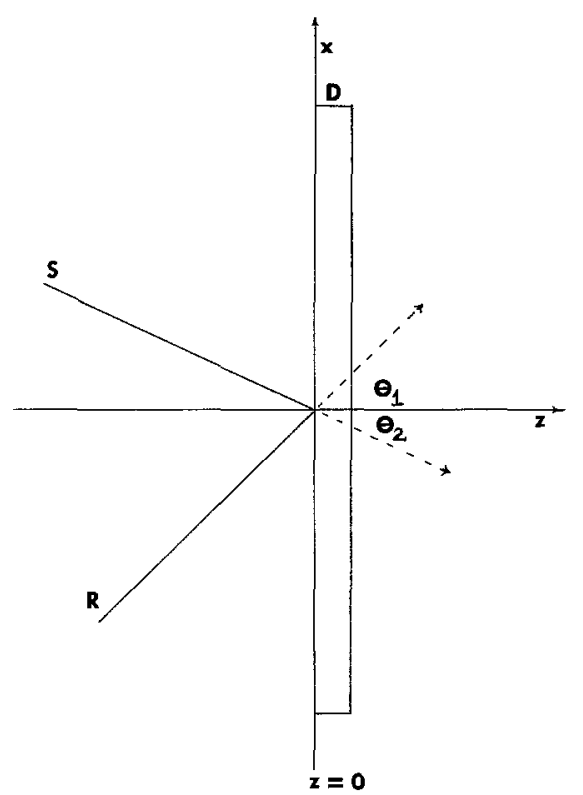

Fig. 1. Construction of single thick grating by interferring plane waves $R$ and $S$. The emulsion thickness is $D$

The film emulsion of thickness $D$ is perpendicular to the $z$ axis and is assumed to be of infinite extent in the $x$ and $y$ directions. We limit the analysis to one lateral dimension. Plane wave $R$ of amplitude $a_{1}$ incident at angle $\theta_{1}$ (angles measured inside the emulsion and the sign convention is that of Goodman [7]) with respect to the $z$ axis and $S$ of amplitude $a_{2}$ at $\theta_{2}$ interfere to produce a grating throughout the volume of the emulsion. The exposure $E=I \tau$ ( $I$ is the total intensity and $\tau$ is the exposure time) can be written as [5]

$E(x, z)=\tau I_{0}\left\{1+r \cos \left[2 \pi f\left(x-x_{z}\right)\right]\right\}$,

where $f=\left(\sin \theta_{1}-\sin \theta_{2}\right) / \lambda, \lambda$ is the free space wavelength, $x_{z}=z \tan \phi, \phi=\left(\theta_{1}+\theta_{2}\right) / 2$ is the slant of the fringes, $I_{0}=a_{1}^{2}+a_{2}^{2}$ and $r=2 a_{1} a_{2} /\left(a_{1}^{2}+a_{2}^{2}\right)$. For any plane $z=$ constant, the fringes are sinusoidally modulated with frequency $f$. However, because of fringe slant (when the construction beam directions are not symmetrical about the $z$ axis) the fringes at $z$ are shifted by $x_{z}$ relative to those at $z=0$ which introduces a relative phase shift of $-2 \pi f x_{z}$. (We stress that $f$ is the frequency of the fringes in any $z=$ constant plane and not the inverse of the perpendicular distance between fringe planes.)

We examine the properties of optical propagation in the thick grating of Fig. 1 by decomposing it into a series of thin slabs of thickness $\Delta z$. The magnitude of $\Delta z$ must be sufficiently small so that each slab acts as a thin grating. A reasonable upper limit on $\Delta z$ to meet this condition is that for the given readout wavelength and grating frequency, Kogelnik's $Q$ factor $\left(Q \sim 2 \pi \lambda \Delta z f^{2}\right)$ [2] for the thin grating be on the order of one. Each of the thin gratings, therefore, exhibits no Bragg effects and its angular distribution of diffracted light is accurately predicted by thin grating theory. We also assume that the actual diffraction process takes place discretely at the planes $z=n \Delta z, n=1,2, \ldots$. To determine the amplitude transmittance of each of the thin gratings that results from the exposure given in (1), we must specify the type of recording material. Here we consider a pure phase material (for example, dichromated gelatin). We also assume a linear relationship between the total index of refraction change $\Delta n$, relative to the bulk emulsion index $n_{0}$, and the exposure $E, \Delta n=m E$. The phase shift resulting from the index change for a plane wave passing through the thin grating at arbitrary angle $\theta_{i}$ is $(2 \pi \Delta n \Delta z) / \lambda \cos \theta_{i}$. Therefore, the amplitude transmittance resulting from the exposure given by (1) for a plane wave at $\theta_{i}$ is [6]

$$
\begin{aligned}
T_{A}(x, z)= & \exp \left[\left(j 2 \pi m \tau I_{0} \Delta z / \lambda \cos \theta_{i}\right)\right. \\
& \left.\cdot\left\{1+r \cos \left[2 \pi f\left(x-x_{z}\right)\right]\right\}\right] .
\end{aligned}
$$

Using a familiar expansion [6], the amplitude transmittance of the $n^{\text {th }}$ grating can be written as

$$
\begin{aligned}
T_{A}(x, n)= & \exp \left\{j 2 \pi m \tau I_{0} \Delta z / \lambda \cos \theta_{i}\right\} \\
& \sum_{q=-\infty}^{\infty}(j)^{q} J_{q}(b) \exp \left[j 2 \pi q f\left(x-x_{n}\right)\right],
\end{aligned}
$$

where $\mathbf{J}_{q}(b)$ is the $q^{\text {th }}$ order Bessel function, $b=\left(2 \pi \Delta z m \tau r I_{0}\right) / \lambda \cos \theta_{i}=\left(2 \pi \Delta z n_{1}\right) / \lambda \cos \theta_{i} \quad$ and $x_{n}=n \Delta z \tan \phi . n_{1}=m \tau r I_{0}$ is the index modulation of the grating. The $n^{\text {th }}$ thin grating can therefore be represented by an infinite superposition of amplitude gratings of spatial frequency $q f$ with diffraction amplitudes given by the Bessel function of order $q$. The exponential outside the summation sign in (3) represents an unimportant common phase factor and will be dropped. Note that the diffraction amplitudes depend upon the thickness of the thin slab and the wavelength and direction of the incident plane wave as well as upon the index modulation $n_{1}$. Also the $q^{\text {th }}$ frequency component is shifted in phase by $-2 \pi q f n \Delta z \tan \phi$ because of fringe slant. The effect of this phase shift is important because it is the means by which angular selectivity about the Bragg angle results. 
To find the effect of the $n^{\text {th }}$ slab upon an incident plane wave of arbitrary spatial frequency we use the results of thin grating theory. Consistent with such theory the effect of each thin slab can be divided into two parts. First, the propagation of the incident wave over the interval $\Delta z$ which is described by the free space transfer function [7]. Second, the separation of the incident wave into the various diffracted waves at $z=n \Delta z$ as dictated by the amplitude transmittance function in (3). Therefore, for an input plane wave of spatial frequency $f_{i}$, amplitude $A_{i}$ and phase $\Phi_{i}$ at $z=(n-1) \Delta z$, the field at $z=n \Delta z$ (i.e., out of the $n^{\text {th }}$ grating) is

$$
\begin{aligned}
A(x, n \Delta z)= & \exp \left(j \Phi_{i}\right) A_{i} \sum_{q=-\infty}^{\infty}(j)^{q} \mathrm{~J}_{q}(b) \\
& \cdot \exp \left(-j 2 \pi q f x_{n}\right) \\
& \cdot \exp \left[\frac{\left(j 2 \pi n_{0} \Delta z\right)}{\lambda}\left(1-\lambda^{2} f_{i}^{2}\right)^{1 / 2}\right] \\
& \cdot \exp \left[j 2 \pi\left(f_{i}+q f\right) x\right] .
\end{aligned}
$$

For each plane wave incident upon the $n^{\text {th }}$ slab there is a countable infinity of plane waves of spatial frequency $\left(f_{i}+q f\right)$ produced. The amplitude of the $q^{\text {th }}$ wave is the product of the input amplitude and the strength of the $q^{\text {th }}$ grating frequency component $\left[\mathrm{J}_{q}(b)\right.$ in this case]. The phase shift of each wave is given by three factors. The term $\exp \left(-2 \pi q f x_{n}\right)$ in (4) represents the phase shift for the $q^{\text {th }}$ order of the grating that results from the lateral shift of the $n^{\text {th }}$ grating. The term $\exp \left\{\left[\left(j 2 \pi n_{0} \Delta z\right) / \lambda\right]\left(1-\lambda^{2} f_{i}^{2}\right)^{1 / 2}\right\}$ gives the spatial frequency dependent phase shift due to propagation over the longitudinal distance $\Delta z$. In addition, because the emulsion is a phase material, there is the phase factor $(j)^{q}$. Because each plane wave output from the $n^{\text {th }}$ slab is input to the $(n+1)$ slab, (4) shows that for an incident plane wave of frequency $f_{i}$ at $z=0$ only plane waves of spatial frequency $\left(f_{i}+q f\right)$ are present inside the thick grating.

Suppose we illuminate the thick grating at $z=0$ with a plane wave of spatial frequency $f_{i}$ and amplitude $A_{i}$. We represent the amplitudes of each of the possible plane waves that exit from the $n^{\text {th }}$ grating by

$$
A_{n}=\left(\begin{array}{c}
A_{0} \\
A_{1} \\
\vdots \\
A_{l} \\
\vdots
\end{array}\right), \quad l \text { integer } \geqq 0
$$

where $A_{l}$ is the total amplitude of the plane wave of spatial frequency $\left[f_{i}-(l+1) f / 2\right]$ for $l$ odd and $\left(f_{i}+l f / 2\right)$ for $l$ even. Because (4) is valid for an input plane wave of arbitrary spatial frequency, we can relate the total amplitude of each of the plane waves at $z=n \Delta z$ to its value at $z=(n-1) \Delta z$ by

$A_{n}={ }_{n} H A_{n-1}$,

where the matrix of coupling coefficients ${ }_{n} H_{k j}$, determined from (4) describe the amplitude and phase shift effected by the $n^{\text {th }}$ thin grating in diffracting the $j^{\text {th }}$ plane wave into the $k^{\text {th }}$ one. ${ }_{n} H_{k j}$ is non zero only if the thin grating has an amplitude frequency component equal to the difference between the input and output spatial frequencies. The functional form of the coupling coefficients which we have derived for a pure phase material, can always be determined from thin grating theory and depend upon the type of gratingphase, amplitude or mixed and upon the assumed relationship between exposure and film response. The subscript $n$ on the coupling matrix emphasizes the fact that because of fringe slant the coupling coefficients depend upon the longitudinal position of the thin grating slab. The coupling matrix for the pure phase case is expressed explicitly in Appendix A. If we now use the initial condition at $z=0$ to write

$A_{0}=\left(\begin{array}{c}A_{i} \\ 0 \\ 0 \\ \vdots\end{array}\right)$,

then the amplitudes at $z=n \Delta z$ are

$A_{n}={ }_{n} H \ldots{ }_{1} H A_{0}$.

The amplitude of each of the plane waves at the exit plane $z=D$ are given by (6) for $n=N=D / \Delta z$.

\section{Results for a Single and Double Grating}

Generally for practical exposure values the argument $b$ of the $\mathrm{J}_{q}(b)$ is sufficiently small so that for large $q$ the $\mathrm{J}_{q}(b)$ are negligibly small, and the $H$ matrices can be truncated with little loss in accuracy. We have written a simple computer program to perform the matrix multiplication of (6). To check the accuracy of the results predicted by this approach for a single grating, we compare our results with those of Kogelnik. Using the matrix theory, Kaspar has shown that Kogelnik's results are accurate for gratings of low modulation and large thickness [4]. We therefore 


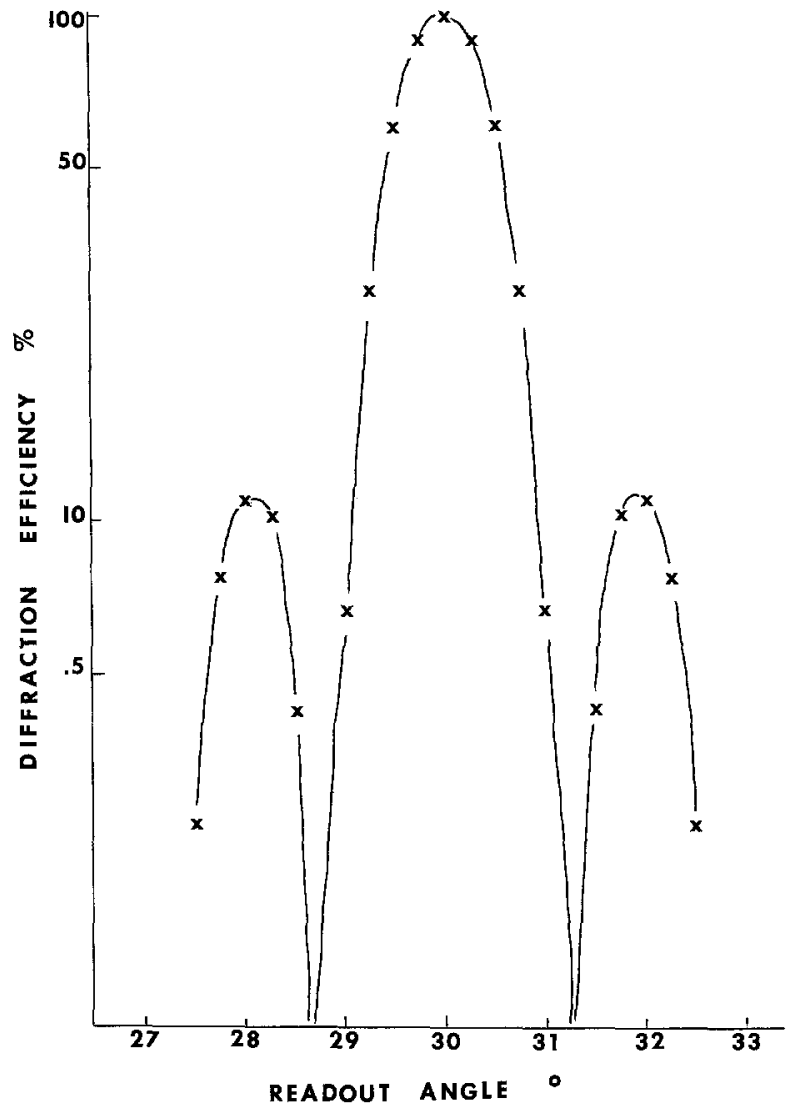

Fig. 2. Diffraction efficiency of the first order versus the angle of the readout beam for single grating. The construction beams are at $0^{\circ}$ and $30^{\circ}, \lambda=0.6328 \mu \mathrm{m}$ and $D=50 \mu \mathrm{m}$

apply this method to a grating constructed on a $50 \mu \mathrm{m}$ emulsion with construction beams at $0^{\circ}$ and $30^{\circ}$ and $\lambda=0.6328 \mu \mathrm{m}$. The index of refraction modulation $n_{1}$ used is that which according to Kogelnik would give $100 \%$ diffraction into the first order for readout at the Bragg angle $\left(30^{\circ}\right)$. In Fig. 2 we plot the calculated diffraction efficiency of the first order versus readout angle. The results are in good agreement with those predicted by Kogelnik for the case of the readout wave polarized perpendicular to the plane of incidence (i.e., along the grating lines). The intensity of the higher orders was found to be essentially zero.

To demonstrate the general applicability of this approach we give some new results for the case of a grating made with two fringe structures. As an interesting case we consider the grating in Fig. 3 made by an incoherent superposition of interferring plane waves $S_{1}$ and $R$ and then $S_{2}$ and $R$. There is no inter-

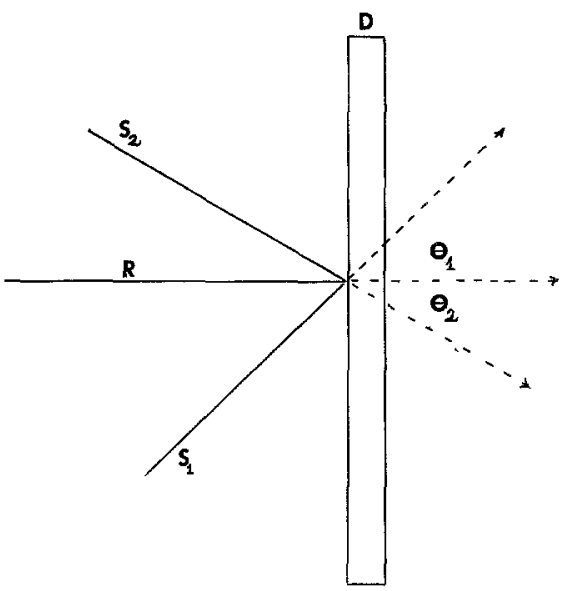

Fig. 3. Construction of double grating structure by interferring plane waves $R$ and $S_{1}$ and then $R$ and $S_{2}$

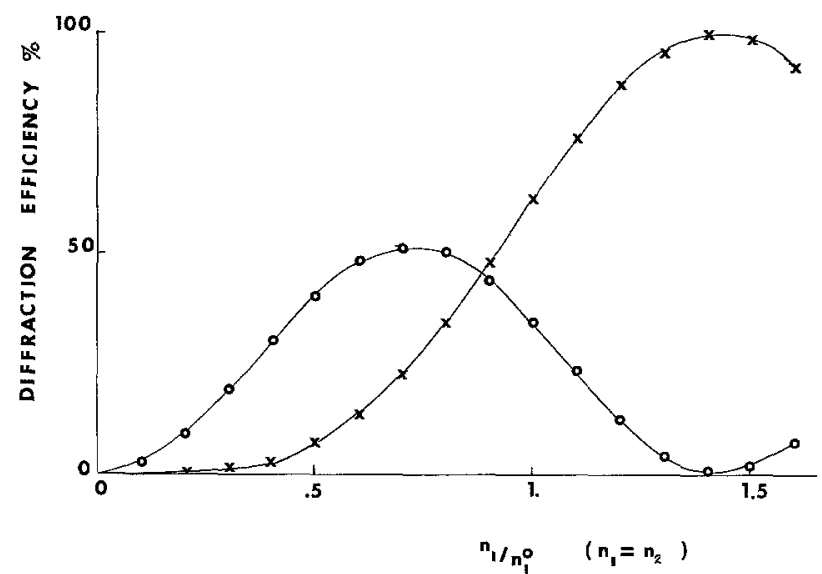

Fig. 4. Diffraction efficiency of the first $(o)$ and cross-coupled $(x)$ orders versus the relative index modulation $n_{1} / n_{1}^{0}$ for a double grating constructed as in Fig. 3. $n_{1}^{0}$ is the index modulation which would give $100 \%$ diffraction efficiency in the first order in the absence of the second grating. The two gratings have equal index modulation, $R$ is in the $z$ direction, $\theta_{1}=30^{\circ}, \theta_{2}=-25^{\circ}, D=24 \mu \mathrm{m}$ and $\lambda=0.6328 \mu \mathrm{m}$

ference between $S_{1}$ and $S_{2}$. There are two distinct but overlapping fringe patterns recorded throughout the volume of the emulsion. Therefore each thin grating has two basic frequency components and corresponding lateral shift factors due to fringe slope as well as higher order harmonics of each basic frequency. The possible plane waves inside the emulsion therefore include those that are diffracted by one fringe structure and then subsequently by the other. The coupling matrices are expanded in a straightforward way to include such coupling. Note that each 
fringe structure has the construction beam $R$ in common. We apply this method for a read-out beam incident at the angle of $S_{1}$. We find as a result that, in addition to a strong diffracted beam in the direction of $R$, there is also a strong diffracted beam in the direction of $S_{2}$. Because the readout beam $S_{1}$ is at the Bragg angle of one fringe structure and its diffracted beam $R$ is at the Bragg angle of the other structure, we might expect this doubly diffracted wave, which we call the cross-coupled order, to become quite
Acknowledgements. I thank Professors E. N. Leith and J. Upatnieks and S. Case for helpful discussions during the course of this work. The generous support of the National Science Foundation (Grant No. GK-42361) is also greatly appreciated.

\section{Appendix A}

We show below the form of ${ }_{n} H$ for a single thick grating in a pure phase material.

$$
{ }_{n} H=\left(\begin{array}{cccc}
e^{j \alpha_{0}} \mathrm{~J}_{0}\left(b_{0}\right) & j e^{j \alpha_{1}} \mathrm{~J}_{1}\left(b_{1}\right) e^{j \delta_{1}(n)} & -j e^{j \alpha_{2}} \mathrm{~J}_{-1}\left(b_{2}\right) e^{-j \delta_{1}(n)} & \ldots \\
-j e^{j \alpha_{0}} \mathrm{~J}_{-1}\left(b_{0}\right) e^{-j \delta_{1}(n)} & e^{j \alpha_{1}} \mathrm{~J}_{0}\left(b_{1}\right) & -e^{j \alpha_{2}} \mathrm{~J}_{-2}\left(b_{2}\right) e^{-j \delta_{2}(n)} & \ldots \\
j e^{j \alpha_{0}} \mathrm{~J}_{1}\left(b_{0}\right) e^{+j \delta_{1}(n)} & -e^{j \alpha_{1}} \mathrm{~J}_{2}\left(b_{1}\right) e^{j \delta_{2}(n)} & e^{j \alpha_{2}} \mathrm{~J}_{0}\left(b_{2}\right) & \ldots
\end{array}\right),
$$

strong. Indeed, it does. In Fig. 4 we plot the diffraction efficiency of the first and cross-coupled orders versus grating index modulation $n_{1}$. Each fringe structure was assumed to have the same index modulation. The cross-coupled order becomes strong at the expense of the first order which would be $100 \%$ for $n_{1} / n_{1}^{0}=1$ if the second fringe structure was not present. The presence of the second grating limits the maximum diffraction efficiency of the first order; however, the cross-coupled order can become $100 \%$ efficient (i.e. all the incident light is diffracted into this order) for sufficiently high index modulation.

This rather interesting result demonstrates the importance of cross-coupling of energy between muliple gratings recorded in the same thick emulsion and rules out the possibility of treating the effect of each grating independently. It also shows the flexibility of the above method in treating optical propagation in multiple grating structures. Other results such as the effect of offsetting one fringe structure with respect to the other (such that they no longer have a common construction beam and cross-coupling is reduced) and of varying the relative modulations of the two structures as well as the angular selectivity curves in such cases will be reported in a subsequent paper.

$$
\begin{aligned}
& \text { where } \\
& \qquad \begin{aligned}
f_{l} & = \begin{cases}f_{i}-(l+1) f / 2 & \text { lodd } \\
f_{i}+l f / 2 & l \text { even }\end{cases} \\
b_{l} & =\frac{2 \pi \Delta z n_{1}}{\lambda \sqrt{1-\lambda^{2} f_{l}^{2}}} \\
\alpha_{l} & =\frac{2 \pi \Delta z n_{0}}{\lambda} \sqrt{1-\lambda^{2} f_{l}^{2}} \\
\delta_{q}(n) & =-2 \pi q f n \tan \phi,
\end{aligned}
\end{aligned}
$$

where $\lambda$ is the free space wavelength of the readout wave, $f_{i}$ is its spatial frequency, $\phi$ is the slope of the fringes with respect to the $z$ axis and $n_{0}$ is the bulk refractive index of the emulsion.

\section{References}

1. E.N.Leith, A.Kozma, J.Upatnieks, J.Marks, N.Massey: Appl. Opt. 5, 1303 (1966)

2. H. Kogelnik: Bell Syst. Tech. J. 48, 2909 (1969)

3. C. B. Burckhardt: J. Opt. Soc. Am. 56, 1502 (1966)

4. F. G. Kaspar: J. Opt. Soc. Am. 63, 37 (1973)

5. R.J.Collier, C.B.Burckhardt, L.H.Lin: Optical Holography, (Academic Press, New York 1971) p. 231

6. J. Upatnieks, C.D.Leonard: IBM J. Res. Devel. 527 (1970)

7. J.W.Goodman: Introduction to Fourier Optics (McGraw-Hill, New York 1968) p. 54 